\title{
A THEOREM ON INTERMEDIATE REDUCIBILITIES
}

\section{T. G. MCLAUGHLIN}

Let $\alpha, \beta$ be two sets of natural numbers. Then [2] the least upper bound of (the Turing degrees of) $\alpha$ and $\beta$ is the (Turing degree of the) set $J(\alpha, \beta)=\{2 x \mid x \in \alpha\} \cup\{2 x+1 \mid x \in \beta\}$. In general, we shall denote by $|\alpha|_{T}$ the Turing degree of a set $\alpha$ of natural numbers, and by $|\alpha|_{M}$ and $|\alpha|_{t t}$ the many-one and truth-table degrees, respectively, of $\alpha$ [5]. It is a trivial fact that $|J(\alpha, \beta)|_{M}$ and $|J(\alpha, \beta)|_{t t}$ are least upper bounds for the pairs $|\alpha|_{M},|\beta|_{M}$ and $|\alpha|_{t t},|\beta|_{t t}$, respectively. We denote by $\leqq_{T}$, $\varliminf_{M}$, and $\leqq_{t t}$ the partial order of degrees in the Turing, many-one, and truth-table semilattices, respectively.

The following fact about the semilattice of Turing degrees is well known and easy to prove:

Proposition. If $\alpha, \beta$ are number sets which can be separated by disjoint recursively enumerable sets $\gamma, \delta$ (thus, $\alpha \subseteq \gamma, \beta \subseteq \delta$, and $\gamma \cap \delta=\varnothing$ ), then $|\alpha \cup \beta|_{T}=|J(\alpha, \beta)|_{T}$.

The object of this note is to exhibit a pair of Theorems (and a Corollary) showing how this Proposition breaks down if we consider finer semilattices than that of the Turing degrees; specifically, if we consider the $\leqq_{M^{-}}$and $\leqq_{t}$-semilattices.

We acknowledge that our discovery of Theorem 1 was the result of brooding over Lachlan's proof, in [3], that $\leqq_{t t}$ differs, on the r.e. sets, from the $\leqq_{w}$ relation of Friedberg and Rogers [1]. Indeed, Lachlan's result is a corollary to Theorem 1 , since, as C. G. Jockusch has pointed out to us, $J(\alpha, \beta) \leqq{ }_{w} \alpha \cup \beta$ whenever $\alpha, \beta$ are disjoint r.e. sets.

THEOREM 1. There exist disjoint r.e. sets $\alpha$ and $\beta$ such that

$$
\alpha \cup \beta{ }_{M} J(\alpha, \beta)
$$

and

$$
\alpha t_{t} \alpha \cup \beta \quad\left(\text { whence } J(\alpha, \beta) \ddagger_{t \imath} \alpha \cup \beta\right) \text {. }
$$

Proof. We employ a priority construction of the elementary variety (finitely many injuries per requirement). Four types of markers are used: $\Lambda_{n}, \Sigma_{n}$, and *. A number $n$ shall be free at a given point in the construction just in case neither $n$ nor any larger number bears

Received by the editors October 30, 1966. 
or has previously borne any type of marker, up to the point in question. " $\alpha$ ", " $\beta$ " denote, respectively, the portions of $\alpha, \beta$ defined by the end of stage $s$; " $c^{8}$ " denotes the characteristic function of $\alpha^{8} \cup \beta^{s}$. Let $T_{0}, T_{1}, T_{2}, \cdots$ be a recursive enumeration of all truth-table conditions. If $c$ is the characteristic function of a set and $\left\langle x_{1}, \cdots, x_{j}\right\rangle$ is the tuple of test numbers associated with condition $T_{k}$, we write " $T_{k}\left(c\left(x_{1}\right), \cdots, c\left(x_{j}\right)\right)=0$ " to mean that $c\left(x_{1}\right), \cdots, c\left(x_{j}\right)$ is a minus row of the table involved in condition $T_{k}$, and " $T_{k}\left(c\left(x_{1}\right), \cdots, c\left(x_{j}\right)\right)$ $=1$ " to mean that $c\left(x_{1}\right), \cdots, c\left(x_{j}\right)$ is a plus row of that table. The construction proceeds as follows:

Stage 0. Set $\alpha^{0}=\beta^{0}=\varnothing$ and go to Stage 1 .

Stage $s, s>0$.

Case I. $(s)_{0}=2 k$. If $s=2^{2 k}$, put marker $\Lambda_{k}$ on the smallest free number. Let $r$ be the number bearing $\Lambda_{k}$. If $r$ also bears a ${ }^{*}$, set $\alpha^{s}=\alpha^{s-1}, \beta^{s}=\beta^{s-1}$, and go on to Stage $s+1$. Otherwise, proceed $s$ steps in the computation of $\phi_{k}(r)$, where $\left\{\phi_{i} \mid i=0,1,2, \ldots\right\}$ is the standard normal-form enumeration of the 1-place partial recursive functions. If no value for $\phi_{k}(r)$ is obtained, set $\alpha^{s}=\alpha^{s-1}, \beta^{s}=\beta^{s-1}$, and go on to Stage $s+1$. Otherwise, consider whether $\phi_{k}(r) \in J\left(\alpha^{s-1}, \beta^{s-1}\right)$. If so, set $\alpha^{s}=\alpha^{s-1}, \beta^{s}=\beta^{s-1}$, place a* on $r$, and proceed to Stage $s+1$. If $\phi_{k}(r) \notin J\left(\alpha^{s-1}, \beta^{s-1}\right)$, two cases arise.

Case A. $\phi_{k}(r)=2 r$. Then set $\alpha^{s}=\alpha^{s-1}, \beta^{s}=\beta^{s-1} \cup\{r\}$, place a ${ }^{*}$ on $r$, and a + on $\phi_{k}(r)$ if the latter is free, move all attached markers $\Lambda_{j}$ and $\Sigma_{m}$ with $j>k$ and $m \geqq k$ down (without disturbing their order relative to one another) from their current positions to the first available free numbers, and go to Stage $s+1$.

Case B. $\phi_{k}(r) \neq 2 r$. Then set $\alpha^{s}=\alpha^{s-1} \cup\{r\}, \beta^{s}=\beta^{s-1}$, place a ${ }^{*}$ on $r$, and a + on $\phi_{k}(r)$ if the latter is free, move markers as in Case A, and go to Stage $s+1$. This completes the description of Case I.

Case II. $(s)_{0}=2 k+1$. If $s=2^{2 k+1}$, attach $\Sigma_{k}$ to the smallest free number. Let $r$ be the position of $\Sigma_{k}$. If $r$ also bears a ${ }^{*}$, set $\alpha^{s}=\alpha^{s-1}$, $\beta^{s}=\beta^{s-1}$, and go to Stage $s+1$. Otherwise, compute $s$ steps in the search for a value for $\phi_{k}(r)$. If no value is obtained, set $\alpha^{s}=\alpha^{s-1}$, $\beta^{s}=\beta^{s-1}$, and go to Stage $s+1$. Suppose, on the other hand, that we find $\phi_{k}(r)=w$. Let $\left\langle x_{1}, \cdots, x_{p}\right\rangle$ be the tuple of numbers involved in $t t$-condition $T_{w}$. If any of $x_{1}, \cdots, x_{p}$ are free, put a + on the largest such. Set $c^{s}=$ the characteristic function of $\alpha^{s-1} \cup \beta^{s-1} \cup\{r\}$. If $T_{w}\left(c^{s}\left(x_{1}\right), \cdots, \quad c^{s}\left(x_{p}\right)\right)=0, \quad$ set $\quad \alpha^{s}=\alpha^{s-1} \cup\{r\}, \quad \beta^{s}=\beta^{s-1}$. If $T_{w}\left(c^{s}\left(x_{1}\right), \cdots, c^{s}\left(x_{p}\right)\right)=1$, set $\alpha^{s}=\alpha^{s-1}$ and $\beta^{s}=\beta^{s-1} \cup\{r\}$. In either case, place a ${ }^{*}$ on $r$ and move all attached markers $\Lambda_{j}$ and $\Sigma_{j}$ with $i>k$ down (without disturbing their order relative to one another) 
from their current positions to the first available free numbers, and go to Stage $s+1$.

This completes the description of Case II and of Stage $s(s>0)$.

We of course set $\alpha=\bigcup_{n} \alpha^{n}, \beta=\bigcup_{n} \beta^{n}$; it is obvious that $\alpha$ and $\beta$ are disjoint r.e. sets. The proof of the theorem is completed by the following three lemmas, whose proofs are routine on the basis of the construction given above:

Lemma 1. $(\forall k)$ (both $\Lambda_{k}$ and $\Sigma_{k}$ achieve final positions).

Lemma 2. $(\forall e)\left(\phi_{e}\right.$ is not a many-one reduction of $\alpha \cup \beta$ to $\left.J(\alpha, \beta)\right)$.

Lemma 3. $(\forall e)\left(\phi_{e}\right.$ is not a tt reduction of $\alpha$ to $\left.\alpha \cup \beta\right)$.

COROllary. There exist disjoint r.e. sets $\alpha, \beta$ such that $\alpha \cup \beta$ $\leqq_{M} J(\alpha, \beta) \& J(\alpha, \beta){ }_{t} \alpha \cup \beta$.

Proof. Let $\alpha, \beta$ be as in the theorem; then $\alpha{ }_{t t} \alpha \cup \beta$. Let $R$ be an infinite recursive subset of $\beta$, and let $f$ be a 1-1 recursive function with range $R$. Let $\beta^{*}=(\beta-R) \cup f(\alpha \cup \beta)$. We claim that $\alpha, \beta^{*}$ have the two properties required in the statement of the corollary. First, it is clear from the definition of $\beta^{*}$ that $\alpha \cup \beta^{*} \leqq{ }_{M} \beta^{*}$; hence, we have $\alpha \cup \beta^{*} \leqq{ }_{M} J(\alpha, \beta)$. Next, it is easy to check that $\alpha \cup \beta^{*} \leqq{ }_{t} \alpha \cup \beta$; this prevents $\alpha$-and so also $J\left(\alpha, \beta^{*}\right)$-from being $t t$-reducible to $\alpha \cup \beta^{*}$.

TheOREM 2. There exist disjoint r.e. sets $\alpha$ and $\beta$ such that $J(\alpha, \beta)$ $\leqq_{M} \alpha \cup \beta$ but $\alpha \cup \beta \${ }_{M} J(\alpha, \beta)$.

Proof. We could deduce Theorem 2 immediately from a result of P. R. Young [6], according to which there exist disjoint, recursively isomorphic, noncreative recursively enumerable sets whose union is creative. However, the proof of Young's theorem is fairly involved, and we prefer a more elementary line of argument. We shall need a simple proposition whose proof appears in [4]: a creative set $\alpha$ can be extended to a creative set $\beta$ in such a way that $\beta-\alpha$ is infinite and devoid of infinite recursively enumerable subsets (i.e., $\alpha$ is simple in $\beta$ ), whereas, if $\alpha, \beta, \gamma$ are any three recursively enumerable sets such that $\beta \cap \gamma=\varnothing, \alpha=\beta \cup_{\gamma},[\delta$ recursively enumerable $\& \delta \cap \beta=\varnothing]$ $\Rightarrow \delta-\alpha$ is recursively enumerable, and [ $\delta$ recursively enumerable $\& \delta \cap \gamma=\varnothing] \Rightarrow \delta-\alpha$ is recursively enumerable, then neither $\beta$ nor $\gamma$ can be extended to a recursively enumerable superset in such a way that the relative difference is infinite but lacks infinite recursive subsets. Moreover, it is known (as a result of close examination by C. E. M. Yates of the Friedberg decomposition procedure for r.e. 
sets) that any nonrecursive, recursively enumerable $\alpha$ can be taken as the $\alpha$ for such a triple $\alpha, \beta, \gamma$. So let $\alpha$ be creative, and let $\alpha, \beta, \gamma$ be such a triple. Then, by the above-cited proposition, neither $\beta$ nor $\gamma$ is creative; hence, since the creative sets constitute the maximal many-one degree for recursively enumerable sets, each of $\beta, \gamma$ is of many-one degree strictly less than $\alpha$. We claim that $\alpha{ }_{M} J(\beta, \gamma)$ holds as well. For if $\alpha \leqq{ }_{M} J(\beta, \gamma)$, then $J(\beta, \gamma)$ is creative. Hence there is another creative set $\delta$ such that $J(\beta, \gamma)$ is simple in $\delta$. Hence either $\{2 x \mid x \in \beta\}$ is simple in $\delta \cap$ the even numbers, or else $\{2 x+1 \mid x \in \gamma\}$ is simple in $\delta \cap$ the odd numbers. The first alternative implies that $\beta$ is simple in a recursively enumerable set, and the second that $\gamma$ is simple in a recursively enumerable set; hence neither can obtain, and our claim is proven.

\section{REFERENCES}

1. R. M. Friedberg and H. Rogers, Jr., Reducibility and completeness for sets of integers, Z. Math. Logik Grundlagen Math. 5 (1959), 117-125.

2. S. C. Kleene and E. L. Post, The upper semilattice of degrees of recursive unsolvability, Ann. of Math. 59 (1954), 379-407.

3. A. H. Lachlan, Some notions of reducibility and productiveness, Z. Math. Logik Grundlagen Math. 11 (1965), 17-44.

4. T. G. McLaughlin, On relative coimmunity, Pacific J. Math. 15, (1965), 13191327.

5. E. L. Post, Recursively enumerable sets of positive integers and their decision problems, Bull. Amer. Math. Soc. 50 (1944), 284-316.

6. P. R. Young, On semicylinders, splinters, and bounded-truth-table reducibility, Trans. Amer. Math. Soc. 115 (1965), 329-339.

Cornell University and

The University of IllinoIs 